\title{
PHẪU THUẬT GLENN HAI HƯỚNG Ở BỆNH NHÂN CÓ HAI TĨNH MẠCH CHỦ TRÊN: CÓ GÌ MỚI VỀ KỸ THUẬT LÀM MIỆNG NỐI?
}

\section{Nguyễn Trần Thuý*, Vũ Xuân Quang*, Đoàn Quốc Hung**, Lê Ngọc Thành*}

\section{TÓM TẮT}

Giới thiệu một kỹ thuật mới trong phẫu thuật Glenn hai hướng có hai tĩnh mạch chủ trên ở bệnh nhân một tâm thất. Một trong những nguyên nhân thất bại của phẫu thuật Glenn hai hướng ở bệnh nhân có hai tĩnh mạch chủ trên là do hai tĩnh mạch này nhỏ, dòng chảy yếu, khi làm miệng nối với động mạch phổi dễ gây xoắn, tắc, huyết khối miệng nối. Việc góp hai tĩnh mạch chủ trên chuyển thành một hợp lưu tĩnh mạch lớn hơn với miệng nối đủ rộng để nối với động mạch phổi có thể giải quyết vấn đề này. Chúng tôi báo cáo 2 trường hợp đã áp dụng thành công kỹ thuật này tại trung tâm tim mạch Bệnh viện $\mathrm{E}$, cung cấp một lưu lượng máu đều đến hai phổi và mang lại sự tăng trưởng kích thước động mạch phổi cũng như giảm thiểu sự hình thành huyết khối.

Tù khóa: Glenn hai hướng, góp hai tĩnh mạch chủ trên.

WHAT'S NEW WITH BILATERAL SUPERIOR VENA CAVA ANASTOMOSIS

IN THE GLENN BIDIRECTIONAL PROCEDURE?

\section{SUMMARY}

We propose a new surgical technique to manage the presence of bilateral superior vena cava in single ventricle patients, a recognized risk factor for both bidirectional Glenn anastomosis and Fontan completion. The idea is to convert two small, peripheral and competing bilateral bidirectional cavopulmonary anastomoses into a single, larger, and centrally located cavopulmonary connection. This technique, used in 2 patients in our institution, provides a symmetrical distribution of pulmonary blood flow and may, in fact, yield growth of the central pulmonary arteries as well as prevent thrombus formation.

Keywords: bidirectional Glenn shunts; unifocalization of bilateral

\section{1. ĐẶT VẤN ĐỀ}

Bất thường tĩnh mạch (TM) hệ thống không còn được coi là yếu tố nguy cơ chính đối với phẫu thuật Glenn hai hướng và Fontan. Tuy nhiên, sự hiện diện của một tĩnh mạch chủ trên (TMCT) bên trái vẫn đặt ra một thách thức khó khăn đến việc

hoàn thành các miệng nối tĩnh mạch chủđộng mạch phổi (TMC-ĐMP) an toàn và hiệu quả [1]. Về phương diện giải phẫu khi có hai TMCT thì đường kính nhỏ của $\mathrm{TMCT}$ sẽ làm giảm lưu lượng máu, dòng chảy không cân bằng có thể gây ra ứ máu. Lưu lượng máu đến hai phổi không đều tạo ra dòng chảy chậm ở hợp lưu động mạch phổi, dẫn đến tăng nguy cơ hình thành huyết khối và sự phát triển không thuận lợi của thân động mạch phổi [2],[3].

* Trung Tâm Tim Mạch - Bệnh viện E

** Truò̀ng Đai hoc Y Hà Nồi

Ngườ chịu trách nhiệm khoa học: PGS.TS Lê Ngọc Thành

Ngày nhận bài: 20/03/2015 - Ngày Cho Phép Đăng: 27/03/2015

Phản Biện Khoa học: PGS.TS. Đặng Ngocc Hùng

PGS.TS. Bùi Đức Phú 
Nhằm mục đích cải thiện dòng chảy TMCT đã có nhiều ý tưởng được đưa ra: tạo ra tĩnh mạch vô danh bằng đoạn mạch nhân tạo Gore-tex [4], góp miệng nối dưới hai TMCT thành một hợp lưu TM [3] hoặc hợp nhất hai TMCT thành một TMCT duy nhất [2], ghép đoạn TMCT bằng đoạn mạch ĐMC đồng loại (aortic homograft conduit) [5].

Trong nghiên cứu này, chúng tôi trình bày sự thay đổi của kỹ thuật làm miệng nối hai TMCT trong phẫu thuật Glenn hai hướng mà có thể tối ưu hóa sự phát triển của các nhánh động mạch phổi và làm giảm nguy cơ hình thành huyết khối. Đồng thời qua đó điểm lại những nghiên cứu đã được thực hiện trên thế giới trong việc thay đổi kỹ thuật làm miệng nối TMC-ĐMP của phẫu thuật Glenn hai hướng có hai tĩnh mạch chủ trên.

\section{2. ĐỐI TƯợNG VÀ PHƯƠNG PHÁP NGHIÊN CÚU}

2.1. Đối tượng: hai trường hợp lâm sàng có chẩn đoán là tim một thất, có hai tĩnh mạch chủ trên, đã được phẫu thuật Glenn hai hướng tại bệnh viện E năm 2014.

2.2. Phương pháp nghiên cứu: Thông báo lâm sàng

\section{KẾT QUẢ NGHIÊN CÚ̉}

\subsection{Bệnh án 1}

-Bệnh nhân nam 11 tháng, nặng 7,4 kg, tím, Sp 02: 67\%, đã phẫu thuật blalock trái lúc 3 tháng tuổi.

-Siêu âm, Thông tim: đảo gốc ĐM, ĐMC xuất phát từ thất phải phía trước, thân ĐMP teo tịt, thông liên thất lớn tim gần như một thất, nhĩ ba buồng, còn ống động mạch nhỏ, xoang vành giãn rộng có TMCT trái, cầu nối blalock trái thông.

-Xét nghiệm công thức máu, hóa sinh máu không có gì đặc biệt.

-Bệnh nhân được tiến hành phẫu thuật: mở ngực đường giữa xương ức, lấy màng tim. Đánh giá thương tổn: ĐMC nằm phía trước xuất phát từ thất phải, ĐMP thân nhỏ nằm ở phía sau, TMCT bên phải nhỏ, TMCT bên trái lớn (ưu thế bên trái), có tĩnh mạch chủ dưới (TMCD) bên phải. Ống ĐM nhỏ $5 \mathrm{~mm}$, cầu nối blalock $\mathrm{T}$ thông. Đo áp lực ĐMP trước mổ $16 \mathrm{mmHg}$.

-Thiết lập hệ thống tuần hoàn cơ thể (THNCT), hạ nhiệt độ, đặt cannula ĐMC, hai TMCT, nhĩ phải. Kẹp ĐMC, bảo vệ cơ tim bằng dung dịch Custodiol. Mở nhĩ phải, mở rộng vách liên nhĩ, cắt màng ngăn nhĩ trái. Cắt rời thân ĐMP, khâu đầu trung tâm, mở rộng chạc ba động mạch phổi. Hai TMCT được cắt rời tại vị trí tiếp nối

với nhĩ và được phẫu tích dài nhất có thể. TMCT trái ngắn có nguy cơ không góp được với TMCT bên phải. Chúng tôi sử dụng màng tim tươi để thực hiện kỹ thuật làm thêm một đoạn mạch bằng màng tim cuộn trên bougie Hegar số 12 chỉ prolene 6.0. Sau đó làm miệng nối đầu tự do TMCT trái với đoạn mạch (hình 1 , trái). Hai đầu tự do của hai TMC trên được sáp nhập với một khoảng cách 2 cm ở giữa, ngay dưới cung động mạch chủ, để tạo thành một thân duy nhất có kích cỡ lớn hơn (miệng nối mới hình chữ $\mathrm{Y}$ ), sau đó kết thúc bằng miệng nối chung vào ngã ba ĐMP sử dụng chỉ prolene 7.0 khâu vắt (hình 1 , phải). 

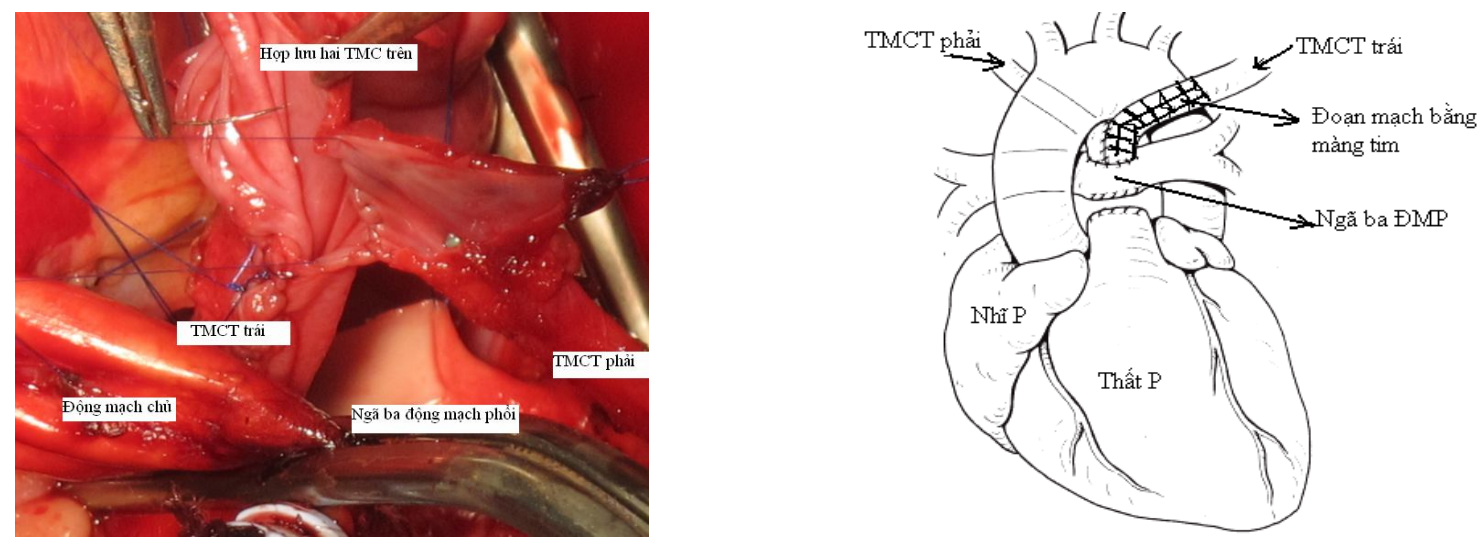

Hình 1: Ống mạch bổ sung bằng màng tim tuơi cuộn nối vào TMCT T (trái) và lược đồ minh họa miệng nối chung vào chạc ba ĐMP (phải)

Sau mổ bệnh nhi ổn định. Siêu âm kiểm tra miệng nối TMC-ĐMP hoạt động tốt, không có chênh áp qua miệng nối, ĐMP giãn tốt cả hai nhánh.

\subsection{Bệnh án 2}

-BN nam, 3 tuổi, $12 \mathrm{~kg}$, vào viện vì tím môi và đầu chi. Khám lâm sàng: sứt môi hở hàm ếch, tím môi, đầu chi, móng tay khum

-Siêu âm: Đảo gốc ĐM, thất phải hai đường ra, thông sàn nhĩ thất toàn bộ, tim dạng một thất, hẹp khít tại van ĐMP

-Thông tim: Đảo gốc ĐM, thông sàn nhĩ thất, hẹp dưới van ĐMP, tim bên phải

Phẫu thuật: Thương tổn trong mổ: ĐMC lớn, quai ĐMC quay trái, có hai TMC trên kích thước bằng nhau, TMCD bên phải, ĐMP hai nhánh tốt, thân ĐMC nhỏ.

Héparine liều không chạy máy ( $1 \mathrm{mg} / \mathrm{kg}$ cân nặng). Cắt rời TMCT phải với nhĩ phải, làm miệng nối TMCT phải với nhánh phải ĐMP. Cắt rời TMCT trái, cuộn màng tim, nối TMCT với đoạn mạch cuộn màng tim. Cắm TMCT trái (đã được kéo dài) với TMCT phải (hình 2).
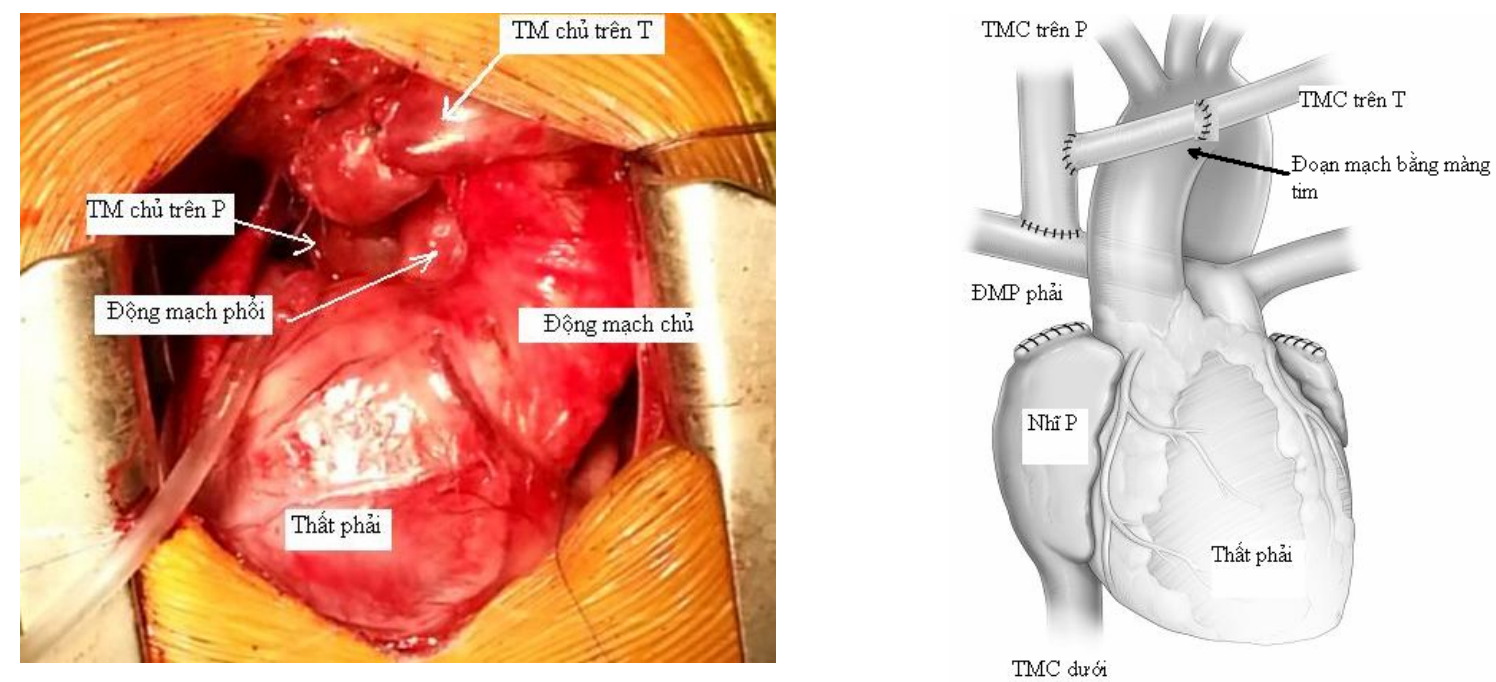

Hình 2: Hình ảnh thương tổn trong mổ (trái) và lược đồ minh họa sau khi hoàn thành các miệng nối (phải) 
-Sau mổ bệnh nhi ổn định. Siêu âm thấy miệng nối TMC-ĐMP hoạt động tốt, không huyết khối, không có chênh áp qua miệng nối, ĐMP giãn tốt cả hai nhánh.

\section{BÀN LUẬn}

Sự hiện diện của hai TMCT thường gặp ở bệnh tim bẩm sinh khác nhau, nhưng nguyên nhân chính xác của sự phát triển bất thường tĩnh mạch hệ thống này vẫn chưa được hiểu rõ. Nghiên cứu giải phẫu cho thấy khi xuất hiện hai TMCT phần lớn kích thước hai TM không đều nhau, sự mất cân bằng về kích thước giải phẫu dẫn đến lưu lượng máu không đều có thể gây ra ứ máu và huyết khối sau khi làm miệng nối với ĐMP trong phẫu thuật Glenn hai hướng [2],[3]. Ngoài ra, một lo ngại là lưu lượng máu từ $\mathrm{TMCD}$ có thể không phân phối đều sau phẫu thuật Fontan, vì có sự cản trở của những luồng dẫn máu từ hai TMCT. Phân phối không đều lượng máu đến phổi có thể dẫn đến dị dạng động tĩnh mạch phổi, tăng nguy cơ hình thành huyết khối và ảnh hưởng đến sự phát triển của hai nhánh ĐMP [3]. Năm 2002 Amodeo và Di Donato đã chỉ rõ hơn những biến đổi của dòng chảy cũng như sự phân luồng máu đến phổi sau phẫu thuật Glenn và Fontan dựa trên những hình ảnh thu được qua chụp cộng hưởng từ và thông tim của 110 bệnh nhân đã phẫu thuật Fontan [6].

Để cải thiện dòng chảy từ hai TMCT đến ĐMP do sự chênh lệch áp lực của dòng máu ở hai TMCT không đều, có nhiều giải pháp kỹ thuật tạo miệng nối được đề ra và công bố trên y văn. Vida VL năm 2006 thông báo một trường hợp 14 tháng tuổi được tạo một $\mathrm{TM}$ vô danh mới bằng một đoạn mạch Gore-tex $5 \mathrm{~mm}$ nối giữa hai TMCT trong phẫu thuật Glenn hai hướng. Tuy thông báo có kết quả tốt nhưng giải pháp dùng mạch nhân tạo để giảm áp dòng chảy của TM vẫn cho nhiều nghi ngại vì khả năng tắc ống cũng như sự phát triển sau này của bệnh nhân [4].

Những nghiên cứu cho thấy lưu lượng máu phổi bị xáo trộn từ TMCT và phân phối lưu lượng máu từ $\mathrm{TMCD}$ là những yếu tố quan trọng trong việc tiên lượng của kết quả phẫu thuật Glenn và Fontan. Năm 2007 Amodeo và Di Donato đã đề xuất một phương pháp mới có khả năng giải quyết một số vấn đề xảy ra ở những bệnh nhân có hai TMCT. Mục đích của phương pháp này là góp hai miệng nối TMCT thành một hợp lưu TMC duy nhất "unifocal" nhằm tăng lưu lượng máu và miệng nối $\mathrm{TMC}$ ĐMP hai chiều nằm ở vị trí ngã ba ĐMP được cho là hiệu quả hơn so với hai miệng nối hai chiều TMC-ĐMP nhỏ, ngoại vi và luôn có sự xung đột giữa các dòng máu dẫn đến lưu lượng dẫn máu tới phổi không đều [3]. Nhìn từ góc độ kỹ thuật, việc hoàn thành các miệng nối TMCĐMP sửa đổi hai chiều này bị ảnh hưởng bởi cung động mạch chủ nếu ĐMC lớn có thể gây căng, thậm chí chèn ép vào hợp lưu hai TMCT, đây cũng là chống chỉ định của phương pháp này. Uu điểm của phương pháp là vị trí hợp lưu hai TMCT có thể điều chỉnh sang phải nhiều hơn một chút so với về phía trái để cho phép đủ bù đắp với các vị trí dự đoán của miệng nối đoạn mạch nhân tạo trong tương lai với TMC dưới trong phẫu thuật Fontan [3].

Với nỗ lực giải quyết vấn đề đường kính hai TMCT không đều về giải phẫu dẫn đến làm giảm lưu lượng máu gây ra ứ máu hình thành huyết khối, không cân bằng sự phát triển kích thước hai ĐMP. Keisuke Nakanishi đề xuất năm 2014 một kỹ thuật phẫu thuật mới đặt tên 
là "thân chung (unifocalization) hai TMCT" [2]. K Nakanishi đưa ra giả thuyết: một thân chung thì lưu lượng máu từ TMC trên kép sẽ phân phối cho cả hai phổi một cách tương tự như phẫu thuật Glenn đơn phương. Phương pháp mới này chỉ áp dụng trong bệnh nhân TMCD và $\mathrm{TMCT}$ lớn hơn và ở cùng một bên, nếu TMCT nhỏ ở cùng bên với TMCD thì thực hiện phẫu thuật Glenn hai hướng thông thường, nếu bệnh nhân có TMCT bằng về kích thước, tác giả chọn $\mathrm{TMCT}$ cùng bên với TMCD làm thân chính. Phẫu thuật được thực hiện cho 9 bệnh nhân, có 1 ca tử vong đầu tiên liên quan đến tắc miệng nối TMCT. Nguyên nhân tử vong được tác giả đưa ra là khi phẫu thuật phát hiện TMCT nhỏ cùng bên với TMCD, tuy nhiên vì để sau này có thể làm phẫu thuật Fontan tác giả vẫn quyết định chọn TMCT nhỏ này làm thân $\mathrm{TM}$ chính. Do nguyên nhân TMCT chính nhỏ hơn so với TMCT khác nên lưu lượng máu tĩnh mạch có thể bị cản trở ở ngã ba của hai $T M C$, điều này dẫn đến tăng áp lực tĩnh mạch trung tâm, phù nề khuôn mặt được quan sát thấy sau phẫu thuật. Nhược điểm của phương pháp vẫn là chiều dài của TMCT có thể quá ngắn để cho phép làm miệng nối tận bên TMC-TMC [2].

Bệnh nhi số 1 của chúng tôi có TMCT bên phải nhỏ cùng phía với $\mathrm{TMCD}$, trong khi đó TMCT bên trái rất lớn chiếm ưu thế, thân ĐMP hẹp đã được biết trước khi phẫu thuật. Việc làm miệng nối TMC-ĐMP, đồng thời mở rộng ngã ba ĐMP cùng một lúc dẫn đến chúng tôi lựa chọn phương pháp tạo hợp lưu TMCT duy nhất tại miệng nối với ngã ba ĐMP. Tuy nhiên khi phẫu tích thấy rằng miệng nối của $\mathrm{TMCT}$ trái không đủ dài để tạo hợp lưu với TMCT phải, để khắc phục vấn đề này chúng tôi dùng giải pháp sử dụng màng tim cuộn. Điều đó không những tạo được một đoạn mạch nhân tạo đủ dài mà còn đủ vật liệu để mở rộng ngã ba ĐMP mà không cần phải xử dụng vật liệu nhân tạo.

Bệnh nhi số 2 của chúng tôi có TMCT bên phải và bên trái cùng kích cỡ, $\mathrm{TMCD}$ nằm bên phải. ĐMC rất lớn nằm quay trái che lấp cả TMCT trái, muốn phẫu tích TMCT trái chúng tôi phải kéo ngược ĐMC sang bên phải. Chúng tôi không sử dụng tim phổi máy vì bệnh nhân này chỉ cần làm miệng nối $\mathrm{TMCT}$ và $\mathrm{OMP}$, tuy nhiên vì có hai TMCT nên không cần phải sử dụng hệ thống giảm áp $\mathrm{TM}$. Sau khi cắt rời TMCT trái, chúng tôi thấy không đủ dài để làm miệng nối tận bên với $\mathrm{TMCT}$ phải, để khắc phục vấn đề này chúng tôi dùng giải pháp màng tim cuộn.

Việc nghiên cứu sử dụng những vật liệu khác nhau nhằm mục đích kéo dài $\mathrm{TMCT}$ để thuận tiện cho việc làm miệng nối TMC-ĐMP đã được thông báo bởi nhiều tác giả [4], [5]. Có thể sử dụng đoạn mạch ghép đồng loại, đoạn mạch nhân tạo, hoặc ghép tự thân sử dụng màng tim cuộn, trong nghiên cứu của chúng tôi việc áp dụng màng tim cuộn để tăng chiều dài TMCT trái là hợp lý nhất đồng thời dùng màng tim để mở rộng ngã ba động mạch phổi.

\section{KẾT LUẬN}

Cần có nhiều bệnh nhân hơn và thời gian nghiên cứu đủ dài để đánh giá hiệu quả của các phương pháp làm miệng nối TMC trong phẫu thuật Glenn hai hướng có hai TMCT trước khi ứng dụng rộng rãi. Tuy nhiên, qua hai bệnh nhân ban đầu này, ít nhất là về mặt lý thuyết, chúng tôi nhận xét giải pháp phẫu thuật góp hai miệng nối TMCT thành một hợp 
lưu TMCT dường như có lợi thế đáng kể so với các kỹ thuật báo cáo khác. Trên thực tế việc phân phối lưu lượng máu đều đến hai phổi máu góp phần tăng trưởng của ĐMP và phòng ngừa hình thành huyết khối. Việc thiếu độ dài của miệng nối $\mathrm{TMC}$ có thể được giải quyết bằng sử dụng màng tim tự thân cuộn làm đoạn mạch tiếp nối.

\section{TÀI LIỆU THAM KHẢO}

1. Iyer GKT et al .Are bilateral superior vena cavae a risk factor for single ventricle palliation? Ann Thorac Surg 2000;70:711—6.

2. Keisuke Nakanishi et al. A new technique for venous unifocalization of the bilateral superior vena cava with the Glenn procedure $\mathbf{J}$ Thorac Cardiovasc Surg 2014;148:356-8
3. Amodeo and Di Donato The Unifocal Bilateral Bidirectional Cavopulmonary Anastomosis. Ann Thorac Surg 2007;84: $2134-5$.

4. Vida VL, Leon-Wyss J, Garcia F, Castañeda AR. A Gore-Tex "new-innominate" vein: a surgical option for complicated bilateral cavopulmonary shunts. Eur J Cardiothorac Surg 2006;29:112-3

5. Chakraborty et al. Use of Aortic Homograft Conduit in Bidirectional Glenn Shunt. Heart, Lung and Circulation. 2007;16:52-54

Amodeo A et al. The beneficial vortex and best spatial arrangement in total cavopulmonary connection. J Thorac Cardiovasc Surg,2002; 124:471-478 